\title{
Serial anthropometry predicts peripheral nerve dysfunction in a community cohort
}

\author{
Kelly R. Ylitalo ${ }^{1,2 *}$ \\ William H. Herman ${ }^{1,2}$ \\ Siobán D. Harlow ${ }^{1}$ \\ ${ }^{1}$ Department of Epidemiology, \\ University of Michigan, \\ Ann Arbor, MI, USA \\ ${ }^{2}$ Department of Internal Medicine, \\ University of Michigan, \\ Ann Arbor, MI, USA \\ *Correspondence to: Kelly R. Ylitalo, \\ 1415 Washington Heights, Ann \\ Arbor, MI 48109-2029, USA. \\ E-mail: kylitalo@umich.edu
}

Received: 20 April 2012

Revised: 19 October 2012

Accepted: 20 October 2012

\begin{abstract}
Background Obesity is a risk factor for glucose intolerance, but the independent role of obesity in the development of peripheral neuropathy is unclear. This study assessed the impact of body size trajectories on prevalent nerve dysfunction in community-dwelling women with and without glucose intolerance.
\end{abstract}

Methods Annual (1996-2008) anthropometric measures of weight, height, waist circumference and body mass index [BMI, weight $(\mathrm{kg}) /$ height $\left(\mathrm{m}^{2}\right)$ ] were assessed in the Study of Women's Health Across the Nation - Michigan site. Glucose intolerance was defined annually on the basis of current use of diabetes medications, self-reported diabetes diagnosis and, when available, fasting glucose. Peripheral nerve dysfunction in 2008 was defined as abnormal monofilament testing or $\geq 4$ symptoms or signs. Linear mixed models were used to determine trajectories of anthropometry by subsequently identified nerve dysfunction status.

Results Mean BMI was $32.4 \mathrm{~kg} / \mathrm{m}^{2}$ at baseline, and $27.8 \%$ of the women had nerve dysfunction in 2008. BMI, weight and waist circumference increased over time. Women who would have nerve dysfunction were significantly larger than women without dysfunction, independent of glucose intolerance. At mean baseline age of 46, BMI, weight and waist circumference differed significantly ( $p$-value $<0.01$ ) by subsequent nerve dysfunction status, independent of glucose intolerance and hypertension. These body size differences were maintained but not exacerbated over time.

Conclusions Peripheral nerve dysfunction is prevalent among communitydwelling women. Twelve years before the nerve assessment, anthropometry differed between women who would and would not have nerve dysfunction, differences that were maintained over time. Obesity deserves attention as an important and potentially modifiable risk factor for peripheral nerve dysfunction. Copyright (C) 2012 John Wiley \& Sons, Ltd.

Keywords peripheral neuropathy; obesity; body size

\section{Introduction}

Currently, two thirds of adults in the United States are overweight or obese [1]. The high prevalence of obesity is a major public health problem because obesity is associated with a variety of chronic diseases, decreased quality of life and premature death $[2,3]$. In particular, obesity is a well-documented risk factor for impaired fasting glucose (IFG) and diabetes mellitus [4,5]. Peripheral nerve dysfunction is a common complication of diabetes mellitus, but the role of obesity in the development of peripheral nerve dysfunction, independent of glucose intolerance, is not well understood [6-8]. 
The pathogenesis of peripheral nerve dysfunction has gained recent attention as an important focus of scientific study [9]. Although diabetic peripheral neuropathy is the most common type of neuropathy, it is estimated that among individuals with diabetes, up to 50\% may have an additional cause of generalized neuropathy [9]. Furthermore, many individuals with diabetes have neuropathy at the time of their diagnosis [10], suggesting that neuropathy may be associated with IFG and may precede, rather than follow, overt type 2 diabetes [11-13]. In addition, the development of peripheral neuropathy in diabetic patients with tight glycemic control suggests a role for risk factors independent of chronic hyperglycemia [14].

Obesity may be an important catalyst in a cascade of factors leading to peripheral nerve damage. Obesity may increase the risk of peripheral nerve dysfunction through IFG $[15,16]$, damaging small nerve fibres as the earliest detectable sign of peripheral neuropathy in glucose deregulation [11,17]. Alternatively, microvascular damage from subclinical cardiovascular abnormalities may also contribute to nerve dysfunction [11]. Recent evidence suggests that ageing, oxidative stress and dyslipidemia, independent of diabetes, may be plausible causal mechanisms [18-20]. Furthermore, obesity may be an important consequence of peripheral nerve dysfunction if individuals with sensory deficits or pain in the lower extremities experience declining levels of physical functioning.

Peripheral nerve dysfunction is an under-appreciated condition that has adverse effects on mobility, physical functioning and quality of life [21]. Assessing the potential role for obesity or body size in the development of peripheral nerve dysfunction is important because obesity can be prevented or managed through education, diet and physical activity. We evaluated trajectories of anthropometric measures and prevalent peripheral nerve dysfunction in a population-based cohort of African American and Caucasian mid-life women with and without glucose intolerance in the United States. We hypothesized that women who had nerve dysfunction would be more obese and have greater changes in their body size over time than women without nerve dysfunction, independent of glucose intolerance.

\section{Materials and methods}

\section{Study population}

The Study of Women's Health Across the Nation (SWAN) is an ongoing population-based longitudinal study of women from seven sites in the United States [22]. This investigation focused on the Michigan SWAN cohort of 543 women (325 African American and 218 Caucasian) selected from two communities near Detroit who in 1996 were aged 42-52 years, had an intact uterus and at least one ovary, reported no current use of hormones and had at least one menstrual period in the prior 3 months. Of the original sample, 418 (77\%) were still active in study year 12 (2008) and eligible to participate in the peripheral neuropathy sub-study. Of these, 396 (95\%) underwent a foot examination and/or completed a symptom questionnaire for peripheral nerve dysfunction. Women who participated in the neuropathy sub-study did not differ by race/ethnicity or baseline age, weight, height, waist and hip circumference, blood pressure and fasting glucose from women who did not participate. The parent and sub-study were approved by the University of Michigan Health Institutional Review Board, and all participants provided written informed consent.

\section{Study variables}

Peripheral nerve dysfunction was assessed using the Michigan Neuropathy Screening Instrument (MNSI) symptom questionnaire [23] and monofilament testing [24] in year 12 (2008) of the study. The MNSI symptom questionnaire is a 15-item questionnaire used to acquire information about the presence (yes/no) of common neuropathy symptoms and signs, including numbness, pain, sensitivity, cramping, prickling feelings, hot/cold differentiation, open sores, dryness, weakness and amputation. Monofilament testing used the placement of a 10-g pre-stressed filament on the dorsal side of the big toe, midway between the nail fold and the distal interphalangeal joint briefly $(<1 \mathrm{~s})$ for ten repetitions. The participant was asked to respond if she felt the filament following each repetition. Peripheral nerve dysfunction was defined as $80 \%$ or fewer correct responses to the brief sensation in either foot [25] or $\geq 4$ symptoms or signs reported on the MNSI questionnaire [26].

A total of 110 women was classified as having peripheral nerve dysfunction: 33 women had abnormal monofilament testing, 57 women reported $\geq 4$ signs or symptoms and 20 women had both. In a preliminary analysis, these two methods identified similar women with respect to body size measurements, so women classified with dysfunction by either method were combined for subsequent analyses. The use of two different instruments to assess the presence of peripheral nerve dysfunction may capture different types and/or sizes of damaged nerves [27].

Age in years was calculated from the interval of birth to follow-up visit date. Race/ethnicity was self-identified at the 1996 baseline as African American or Caucasian. Annual (baseline through year 12) anthropometric measures were collected from each participant. Height was measured without shoes in centimetres by fixed stadiometer. Weight was measured in light clothing in kilogrammes by balance beam scale. Body mass index (BMI) was calculated as weight $(\mathrm{kg}) /$ height $\left(\mathrm{m}^{2}\right)$. Waist and hip circumferences were measured in centimetres by a non-stretching tape measure. BMI, weight and waist circumference were identified a priori as the primary obesity measures of interest for our investigation. BMI $\geq 30 \mathrm{~kg} / \mathrm{m}^{2}$ and waist circumference $\geq 88 \mathrm{~cm}$ were used as risk cut points to identify anthropometric obesity.

Blood pressure (mmHg) was measured according to a standard protocol using a mercury sphygmomanometer following an initial minimum $5 \mathrm{~min}$ resting period. 
Participants were classified as hypertensive if their average systolic blood pressure was $\geq 140 \mathrm{mmHg}$, average diastolic blood pressure was $\geq 90 \mathrm{mmHg}$ or if they reported current use of hypertension treatment.

Fasting blood samples were collected from participants, and fasting glucose was assessed at baseline and in study visit (V) 1, V3, V4, V5, V6, V7 and V12. For this sub-study, samples were assayed for blood glucose $(\mathrm{mg} / \mathrm{dL})$ at the Michigan Diabetes Research and Training Center in Ann Arbor, MI, USA. In V12 (2008), the inter-assay coefficient of variation was $3.6 \%$ at $92 \mathrm{mg} / \mathrm{dL}$ and $2.8 \%$ at $310 \mathrm{mg} / \mathrm{dL}$; the intra-assay coefficient of variation was $2.0 \%$ at 84 and $283 \mathrm{mg} / \mathrm{dL}$. For each study visit, diabetes mellitus status was defined using fasting glucose $\geq 126 \mathrm{mg} / \mathrm{dL}$ when available and/or affirmative responses to annual questions about diagnosed diabetes or current use of antidiabetic medications. Women without diabetes were further classified as having IFG (100-125 mg/dL) or normal fasting glucose $(<100 \mathrm{mg} / \mathrm{dL})$. In addition, a three-level cumulative longitudinal status variable was created for ever-diabetes, ever-IFG but never diabetes and always-normal fasting glucose during the study period.

\section{Statistical analysis}

First, means \pm standard deviation (SD) and frequencies were calculated to quantify baseline study population characteristics. We evaluated the overall prevalence of peripheral nerve dysfunction and the prevalence by categories of cumulative glucose tolerance status. Chi-square tests or student's $t$-tests were used to compare baseline population characteristics between women with and without prevalent peripheral nerve dysfunction in study year 12 .

Next, multivariable logistic regression was used to determine the association between anthropometric measures and peripheral nerve dysfunction in year 12. Model 1 evaluated the association between baseline BMI and nerve dysfunction, adjusted for baseline age, race/ethnicity, baseline hypertension and baseline glucose tolerance status. Model 2 substituted baseline weight for BMI, and Model 3 substituted baseline waist circumference. Models 2 and 3 also added height as a covariate because of its association with neuropathy [28].

Third, we evaluated how BMI $\left(\mathrm{kg} / \mathrm{m}^{2} \pm \mathrm{SD}\right)$, weight $(\mathrm{kg} \pm \mathrm{SD})$ and waist circumference $(\mathrm{cm} \pm \mathrm{SD})$ changed from baseline to year 12 . Student's $t$-tests were used to compare baseline and year 12 anthropometric measures between women with and without nerve dysfunction in year 12 .

Fourth, we compared the prevalence of nerve dysfunction and other characteristics in year 12 between women who were and were not obese in year 12 according to BMI and waist circumference risk cut points. Chi-square tests and student's $t$-tests were used to generate $p$-values.

Finally, linear mixed models (PROC MIXED) with random intercepts and slopes for age were used to measure trajectories of anthropometry over time by subsequent peripheral nerve dysfunction status. BMI, weight and waist circumference were log-transformed to meet model assumptions of homoscedasticity and then back-transformed to the geometric mean of their original scale for graphical and numerical presentation. The BMI model was adjusted for cumulative glucose tolerance status and hypertension, and the weight and waist models were adjusted for cumulative glucose tolerance status, hypertension and height. SAS (version 9.3) was used for all data management and analysis. Statistical tests were two-sided with the level of significance defined as $p$-value $<0.05$.

\section{Results}

Average age at baseline was 46.2 years. The study population was 40\% Caucasian and 60\% African American, by design. At baseline, mean BMI was $32.4 \mathrm{~kg} / \mathrm{m}^{2}$, mean weight was $86.4 \mathrm{~kg}$, mean waist circumference was $94.4 \mathrm{~cm}, 8.3 \%$ of the participants had diabetes, $24.0 \%$ had IFG and $26.5 \%$ were hypertensive.

Overall, $27.8 \%$ of the women had peripheral nerve dysfunction in study year 12 . The prevalence of nerve dysfunction increased across categories of cumulative glucose tolerance status: $21.3 \%$ of the women with normal glucose tolerance had nerve dysfunction, $26.4 \%$ of the women with ever-IFG had nerve dysfunction and $36.6 \%$ of the women with ever-diabetes had nerve dysfunction.

At baseline, women who would have peripheral nerve dysfunction in study year 12 already had significantly larger body sizes than women who would not have neuropathy. In addition, women with nerve dysfunction in study year 12 were more likely to have glucose intolerance at baseline compared with women who did not have nerve dysfunction in study year 12 (Table 1).

In multivariable models adjusted for age, race/ethnicity, hypertension and glucose intolerance, baseline BMI ( $p$-value $=0.01)$ was a significant predictor of having peripheral nerve dysfunction 12 years later. Baseline weight $(p$-value $=0.002)$ and waist circumference $(p$-value $=0.0004)$, independent of height, were also significant predictors of peripheral nerve dysfunction in study year 12 (Table 2).

In the total study population, increases in average BMI, weight and waist circumference were observed during the course of the study. Between baseline and study year 12, average BMI increased from 32.4 to $34.0 \mathrm{~kg} / \mathrm{m}^{2}$, average weight increased from 86.4 to $89.9 \mathrm{~kg}$ and average waist circumference increased from 94.5 to $101.3 \mathrm{~cm}$. Baseline and year 12 body size measurements differed significantly between women with and without peripheral nerve dysfunction in study year 12 .

In study year $12,63.0 \%$ of the study population had a BMI $\geq 30 \mathrm{~kg} / \mathrm{m}^{2}$, and these women were more likely to have peripheral nerve dysfunction, a waist circumference $\geq 88 \mathrm{~cm}$, hypertension and glucose intolerance. In study year $12,75.6 \%$ of the study population had a waist circumference $\geq 88 \mathrm{~cm}$, and these women were more likely to have a BMI $\geq 30 \mathrm{~kg} / \mathrm{m}^{2}$, hypertension and glucose intolerance. They were also more likely to have 
Table 1. Baseline (1996) characteristics of women by peripheral nerve dysfunction status in 2008, Michigan SWAN

\begin{tabular}{lcccc}
\hline & Total $(n=396)$ & PND $(n=110)$ & No PND $(n=286)$ & $p$-value \\
\hline Age, years (SD) & $46.2(2.7)$ & $46.3(2.6)$ & $46.1(2.8)$ & 0.39 \\
Race/ethnicity, \% & & & & \\
$\quad$ Caucasian & 40.4 & 40.9 & 50.2 & 0.90 \\
$\quad$ African American & 59.6 & 59.1 & $83.8(20.4)$ & $<0.0001$ \\
Weight, kg (SD) & $86.4(21.9)$ & $94.2(23.8)$ & $163.0(6.1)$ & 0.04 \\
Height, cm (SD) & $163.4(6.1)$ & $164.4(6.1)$ & $31.4(7.7)$ & $<.0002$ \\
Body mass index, kg/m2 (SD) & $32.4(8.1)$ & $34.9(8.5)$ & $92.0(15.8)$ & 0.0001 \\
Waist, cm (SD) & $94.4(17.3)$ & $100.9(19.3)$ & $112.3(14.8)$ & $0.82(0.07)$ \\
Hip, cm (SD) & $114.3(16.2)$ & $119.5(18.5)$ & 24.8 & 0.005 \\
Waist-to-hip ratio & $0.82(0.07)$ & $0.84(0.08)$ & 72.0 & 0.22 \\
Hypertension, \% & 26.5 & 30.9 & 21.7 & 0.003 \\
Diabetes status, \% & & & 6.3 & \\
$\quad$ Normal & 67.4 & 55.5 & 30.0 & \\
$\quad$ Impaired fasting glucose & 24.0 & 14.6 & & \\
Diabetes & 8.6 & & & \\
\hline
\end{tabular}

SWAN, Study of Women's Health Across the Nation; PND, peripheral nerve dysfunction; SD, standard deviation.

Table 2. Multivariable associations (odds ratio $(95 \% \mathrm{CI})$ ) between baseline measures and peripheral nerve dysfunction in year 12 , Michigan SWAN

\begin{tabular}{|c|c|c|c|c|c|c|}
\hline \multirow[b]{2}{*}{ Baseline measures } & \multicolumn{2}{|c|}{ Model 1} & \multicolumn{2}{|c|}{ Model 2} & \multicolumn{2}{|l|}{ Model 3} \\
\hline & OR $(95 \% \mathrm{Cl})$ & $p$-value & OR $(95 \% \mathrm{Cl})$ & $p$-value & OR $(95 \% \mathrm{Cl})$ & $p$-value \\
\hline Age, years & $1.04(0.96,1.13)$ & 0.36 & $1.06(0.97,1.15)$ & 0.21 & $1.05(0.96,1.14)$ & 0.27 \\
\hline \multicolumn{7}{|l|}{ Race/ethnicity } \\
\hline Caucasian (reference) & 1.00 & & 1.00 & & 1.00 & \\
\hline African American & $0.98(0.61,1.57)$ & 0.93 & $0.93(0.57,1.51)$ & 0.77 & $0.96(0.59,1.56)$ & 0.86 \\
\hline $\mathrm{BMI}, \mathrm{kg} / \mathrm{m}^{2}$ (per 5 units) & $1.24(1.06,1.44)$ & 0.01 & - & & - & \\
\hline Weight, kg (per 5 units) & - & & $1.09(1.03,1.16)$ & 0.002 & - & \\
\hline Height, cm & - & & $1.04(1.00,1.08)$ & 0.09 & $1.04(1.00,1.08)$ & 0.03 \\
\hline Waist circumference, $\mathrm{cm}$ (per 5 units) & - & & - & & $1.14(1.06,1.22)$ & 0.0004 \\
\hline Hypertension (yes vs no) & $1.01(0.59,1.72)$ & 0.96 & $1.07(0.62,1.83)$ & 0.81 & $1.02(0.60,1.76)$ & 0.94 \\
\hline \multicolumn{7}{|l|}{ Diabetes status } \\
\hline Normal (reference) & 1.00 & & 1.00 & & 1.00 & \\
\hline Impaired fasting glucose & $1.45(0.84,2.50)$ & 0.18 & $1.37(0.79,2.38)$ & 0.20 & $1.27(0.73,2.20)$ & 0.41 \\
\hline Diabetes & $2.09(0.96,4.58)$ & 0.07 & $2.22(1.00,4.90)$ & 0.05 & $2.03(0.92,4.48)$ & 0.09 \\
\hline
\end{tabular}

SWAN, Study of Women's Health Across the Nation; BMI, body mass index; OR, odds ratio; Cl, confidence interval.

Model 1 uses baseline BMI as the body size measure, Model 2 substitutes baseline weight and height for BMI and Model 3 substitutes baseline waist circumference and height for BMI.

peripheral nerve dysfunction, but the difference was not statistically significant.

In the mixed model analysis at the mean baseline age of 46 years, we observed significantly higher BMI ( $p$-value $=$ 0.003 ), waist circumference ( $p$-value $=0.001$ ) and weight ( $p$-value $=0.001)$ in women with peripheral nerve dysfunction in study year 12, independent of cumulative glucose intolerance status and hypertension. However, although mean obesity changed over time, body size differences between women who would and would not have nerve dysfunction were maintained over time, and we observed no differences in annual percent increase in obesity by peripheral nerve dysfunction status. On average, BMI increased by $0.34 \%$ ( $95 \%$ confidence interval (CI): 0.14 , 0.54) per year for women who had nerve dysfunction in year 12 and by $0.35 \%$ (95\% CI: $0.23,0.48$ ) for women who did not have nerve dysfunction in year 12 . On average, waist circumference increased by $0.61 \%$ (95\% CI: 0.45 , 0.76) per year for women who had nerve dysfunction in year 12 and by $0.60 \%$ (95\% CI: $0.50,0.69$ ) for women who did not have nerve dysfunction in year 12 . On average, weight increased by $0.29 \%$ (95\% CI: $0.09,0.49)$ per year for women who had nerve dysfunction in year 12 and by 0.32\% (95\% CI: 0.20, 0.45) for women who did not have nerve dysfunction in year 12 . The trajectories of body sizes over time by peripheral nerve dysfunction status in year 12 are depicted in Figure 1(A-C).

\section{Discussion}

To our knowledge, this is the first investigation of the prevalence of peripheral nerve dysfunction and trajectories of body size in a population-based cohort of community-dwelling women. We found significant differences in BMI, waist circumference and weight between women with peripheral nerve dysfunction and women without peripheral nerve dysfunction 12 years before the assessment of peripheral nerve dysfunction, differences that persisted over time. Notably, these differences were present independent of glucose intolerance, suggesting a possible independent role for obesity or obesity-related factors in the aetiology of nerve dysfunction. Trajectories 

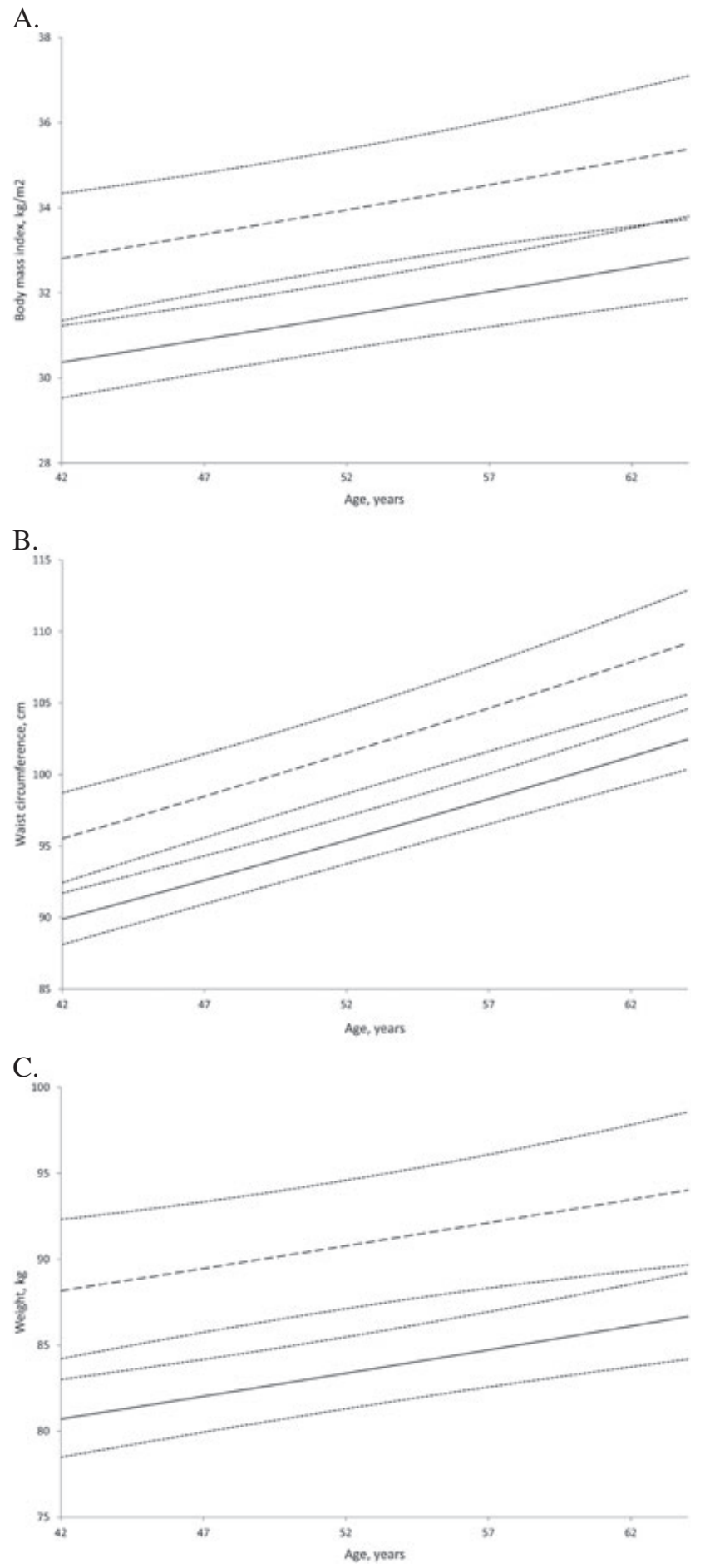

Figure 1. Predicted trajectories of body mass index (A), waist circumference (B) and weight (C) by peripheral nerve dysfunction status in 2008, adjusted for cumulative glucose tolerance status and hypertension (A) or cumulative glucose tolerance status, hypertension and height (B and C), Michigan Study of Women's Health Across the Nation. Large dashed lines are trajectories for peripheral nerve dysfunction, solid lines are trajectories for women without peripheral nerve dysfunction and small dashes are $95 \%$ confidence intervals

of body size measurements were approximately parallel, suggesting that women who would have peripheral nerve dysfunction did not get obese at a faster rate than women without nerve dysfunction.
Our findings that anthropometric measurements are associated with peripheral nerve dysfunction are consistent with other studies. Among individuals with diabetes, BMI has been demonstrated to be a significant predictor of incident neuropathy, independent of diabetes duration and glucose control [14,29]. Two cross-sectional analyses from the National Health and Nutrition Examination Survey found a significant association between anthropometry and peripheral neuropathy measured with monofilament testing. One study reported that weight was significantly associated with neuropathy in persons without diabetes, independent of height, age and glycated haemoglobin A1c [28]. Another study reported that obesity (BMI $\geq 30 \mathrm{~kg} / \mathrm{m}^{2}$ ) and the clustering of abnormal cardiovascular risk factors were positively associated with neuropathy in US adults [30].

The mechanism by which obesity confers a greater risk of peripheral neuropathy is not entirely clear. Adiposity is associated with age, increased oxidative stress, proinflammatory cytokines and dyslipidemia [31-33], which may damage peripheral nerves even in the absence of hyperglycemia [18-20]. Future research should assess the role of specific metabolic products of adipose tissue with respect to neuropathy aetiology because anthropometric measures may be an incomplete proxy of the metabolic environment of obesity [15], particularly considering the proportion of metabolically unhealthy but non-obese individuals in the United States [34].

Nevertheless, BMI, waist circumference and weight are important indicators of an individual's metabolic environment. We found that women who were obese according to anthropometric risk cut points were also more likely to have hypertension and glucose intolerance. BMI and waist circumference in particular correlate well to body composition values, and the use of waist circumference over waist-to-hip ratio is gaining support as a simpler alternative to identify adiposity $[15,35,36]$. BMI and waist circumference are also highly correlated with abnormal metabolic values, over and above measures of percent body fat mass [37]. Although we did not have complete longitudinal measures of triglycerides and cholesterol in this population over time, women with peripheral nerve dysfunction were more likely to report use of lipid-lowering agents (32.7\% vs 21.7\%; p-value $=0.02)$ 2 years prior to our assessment of nerve dysfunction, lending possible support for a dyslipidemia mechanism.

Our measurement of peripheral nerve dysfunction was conducted 12 years after cohort enrollment. We hypothesized that obesity would both cause nerve dysfunction and increase as a result of nerve dysfunction. However, we found no difference in the trajectories (i.e. slopes) of body size measures by nerve dysfunction status. Either peripheral nerve dysfunction developed late during the course of the study and did not have a chance to change the trajectory of obesity or obesity caused nerve dysfunction but did not change as a result of neuropathy. Given the low likelihood that many of the study participants had nerve dysfunction at baseline and the parallel anthropometric trajectories of women with and without nerve dysfunction, it is unlikely that peripheral nerve dysfunction 
caused obesity in this population. However, future studies should establish the timing and causal relationship between obesity and peripheral nerve dysfunction. Furthermore, two thirds of our study population was obese. Future studies may wish to evaluate which correlates of obesity are most strongly associated with nerve dysfunction so that targeted interventions can be developed, particularly in more heterogeneous populations with respect to body size.

Neuropathy has many possible causes [18]. Our assessment of nerve dysfunction was limited to simple, noninvasive measures appropriate for an observational cohort and did not represent a definitive diagnosis of peripheral neuropathy. We were unable to distinguish between the different mechanisms by which obesity may cause nerve dysfunction or eliminate all other causes of peripheral nerve dysfunction. Age-related declines in peripheral nerve function are well documented [20,38,39]. Additional causes of neuropathy include exposure to toxins, vitamin B12 deficiency and alcohol abuse. We would expect toxin exposure to occur randomly, independent of obesity, and would therefore not confound the association between obesity and nerve dysfunction. Although we cannot rule out vitamin B12 malabsorption, no statistically significant differences in vitamin B12 dietary and supplement intake by nerve dysfunction were observed 3 years prior to the assessment of nerve dysfunction, and less than $5 \%$ of our participants reported excessive alcohol consumption (i.e. $\geq 1$ drink/day).

Peripheral neuropathy symptoms, including pain, may interfere with mobility and quality of life $[20,40]$. In a small study of subjects with impaired glucose tolerance and neuropathy, diet and exercise education to improve impaired glucose tolerance also lowered BMI and neuropathic pain [41]. Clearly, neuropathy treatment should target the underlying cause to prevent or slow the progression of the disease $[42,43]$. BMI, waist circumference and weight are easy to measure and readily available to the patient and clinician alike. Identifying easy-to-measure and modifiable risk factors for chronic diseases is an important epidemiologic endeavour, particularly as our population ages. Clinicians who attend obese patients may wish to employ peripheral nerve dysfunction testing because anthropometric measures and their correlates may prove to be important targets for interventions to prevent neuropathy or improve pain. responsibility for the integrity of the data and the accuracy of the data analysis. No potential conflicts of interest relevant to this article are reported.

Dr. Herman's effort was supported in part by grants from the National Institute of Diabetes and Digestive and Kidney Diseases [Grants P60DK020572 (MDRTC) and P30DK092926 (MCDTR)].

The SWAN has grant support from the National Institutes of Health (NIH), DHHS, through the National Institute on Aging (NIA), the National Institute of Nursing Research (NINR) and the NIH Office of Research on Women's Health (ORWH) [Grants NR004061 and AG012505, AG012535, AG012531, AG012539, AG012546, AG012553, AG012554, AG012495 and AG017719 (SWAN Repository)]. The content of this article is solely the responsibility of the authors and does not necessarily represent the official views of the NIA, NINR, ORWH or the NIH.

Clinical centres: University of Michigan, Ann Arbor - Siobán Harlow, PI 2011-present; MaryFran Sowers, PI 1994 2011; Massachusetts General Hospital, Boston, MA - Joel Finkelstein, PI 1999-present; Robert Neer, PI 1994 1999; Rush University, Rush University Medical Center, Chicago, IL - Howard Kravitz, PI 2009-present; Lynda Powell, PI 1994-2009; University of California, Davis/ Kaiser - Ellen Gold, PI; University of California, Los Angeles - Gail Greendale, PI; Albert Einstein College of Medicine, Bronx, NY - Carol Derby, PI 2011-present; Rachel Wildman, PI 2010-2011; Nanette Santoro, PI 2004-2010; University of Medicine and Dentistry New Jersey Medical School, Newark - Gerson Weiss, PI 1994-2004; and the University of Pittsburgh, Pittsburgh, PA - Karen Matthews, PI.

$\mathrm{NIH}$ program office: National Institute on Aging, Bethesda, MD - Sherry Sherman 1994-present; Marcia Ory 1994-2001; National Institute of Nursing Research, Bethesda, MD - program officers.

Central laboratory: University of Michigan, Ann Arbor Daniel McConnell (Central Ligand Assay Satellite Services). SWAN repository: University of Michigan, Ann Arbor Dan McConnell 2011; MaryFran Sowers 2000-2011.

Coordinating centre: University of Pittsburgh, Pittsburgh, PA - Kim Sutton-Tyrrell, PI 2001-present; New England Research Institutes, Watertown, MA - Sonja McKinlay, PI 1995-2001.

Steering committee: Susan Johnson, current chair; Chris Gallagher, former chair.

We thank the study staff at each site and all the women who participated in SWAN.

\section{Acknowledgements}

KRY wrote the manuscript and analyzed the data. SDH and WHH contributed to the discussion and reviewed and edited the manuscript. KRY had full access to all of the data in the study and takes

\section{Conflict of interest}

No relevant financial conflicts of interest.

\section{References}

1. Wang Y, Beydoun MA. The obesity epidemic in the United States-gender, age, socioeconomic, racial/ethnic, and geographic characteristics: a systematic review and meta-regression analysis. Epidemiol Rev 2007; 29: 6-28.
2. U.S. Department of Health and Human Services. The Surgeon General's call to action to prevent and decrease overweight and obesity. U.S. Department of Health and Human Services, Public Health Service, Office of the Surgeon
General: Rockville, MD, 2001. Available from: U.S. GPO, Washington.

3. Jia H, Lubetkin EI. Obesity-related qualityadjusted life years lost in the U.S. from 1993 to 2008. Am J Prev Med 2010; 39(3): 220-227. 
4. Vazquez G, Duval S, Jacobs DR, Silventoinen K. Comparison of body mass index, waist circumference, and waist/hip ratio in predicting incident diabetes: a meta-analysis. Epidemiol Rev 2007; 29: 115-128.

5. Deshpande AD, Harris-Hayes M, Schootman M. Epidemiology of diabetes and diabetes-related complications. Phys Ther 2008; 88(11): 1254-1264.

6. Miscio G, Guastamacchia G, Brunani A, Priano L, Baudo S, Mauro A. Obesity and peripheral neuropathy risk: a dangerous liaison. J Peripher Nerv Syst 2005; 10: 354-358.

7. Ziegler $\mathrm{D}$, Rathman $\mathrm{W}$, Dickhaus $\mathrm{T}$, Meisinger C, Mielck A, KORA Study Group. Prevalence of polyneuropathy in prediabetes and diabetes is associated with abdominal obesity and macroangiopathy. Diabetes Care 2008; 31(3): 464-469.

8. De Block CEM, Leeuw IM, Van Gaal LF. Impact of overweight on chronic microvascular complications in type 1 diabetic patients. Diabetes Care 2005; 28(7): 1649-1655.

9. Freeman R. Not all neuropathy in diabetes is of diabetic etiology: differential diagnosis of diabetic neuropathy. Curr Diab Rep 2009; 9(6): 423-431.

10. Tesfaye S, Boulton AJ (eds). Diabetic Neuropathy. Oxford University Press: Oxford, 2009.

11. Singleton JR, Smith AG, Russell JW, Feldman EL. Microvascular complications of impaired glucose tolerance. Diabetes 2003; 52: 2867-2873.

12. Gordon Smith A, Robinson Singleton J. Idiopathic neuropathy, prediabetes and the metabolic syndrome. $J$ Neurol Sci 2006; 242: 9-14.

13. Smith AG, Rose K, Singleton JR. Idiopathic neuropathy patients are at high risk for metabolic syndrome. $J$ Neurol Sci 2008; 273: 25-28.

14. Tesfaye S, Chaturvedi N, Eaton SEM, et al. Vascular risk factors and diabetic neuropathy. New Engl J Med. 2005; 352(4): 341-350.

15. Grundy SM, Brewer HB Jr, Cleeman JI, Smith SC Jr, Lenfant C, American Heart Association, National Heart, Lung, and Blood Institute. Definition of metabolic syndrome: report of the National Heart, Lung, and Blood Institute/American Heart Association Conference on Scientific Issues Related to Definition. Circulation 2004; 109: 433-438.

16. Gallagher EJ, LeRoith D, Karnieli E. The metabolic syndrome - from insulin resistance to obesity and diabetes. Med Clin N Am 2011; 95(5): 855-873.

17. Singleton JR, Smith AG, Russell J, Feldman EL. Polyneuropathy with impaired glucose tolerance: implications for diagnosis and therapy. Curr Treat Options Neurol 2005; 7(1): 33-42

18. Smith AG. Impaired glucose tolerance and metabolic syndrome in idiopathic neuropathy. J Peripher Nerv Syst 2012; 17(Supplement): 15-21.

19. Singer MA, Vernino SA, Wolfe GI Idiopathic neuropathy: new paradigms, new promise. $J$ Peripher Nerv Syst 2012; 17(Supplement): 43-49.

20. Di Iorio A, Cherubini A, Volpato S, et al. Markers of inflammation, vitamin $\mathrm{E}$ and peripheral nervous system function: the InCHIANTI study. Neurobiol Aging 2006; 27(9): 1280-1288.

21. Van Schie CHM. Neuropathy: mobility and quality of life. Diabetes Metab Res Rev 2008; 24(Supp 1): S45-S51.

22. Sowers MF, Crawford S, Sternfeld B, et al. SWAN: a multi-center, multi-ethnic, community-based cohort study of women and the menopause. In Menopause: Biology and Pathobiology, Lobo R, Kelsey J, Marcus R (eds). Academic Press: San Diego, 2000; 175-178.

23. Feldman EL, Stevens MJ. Clinical testing in diabetic peripheral neuropathy. Can $J$ Neurol Sci 1994; 21: 4-S3-4-S7.

24. Mueller MJ. Identifying patients with diabetes mellitus who are at risk for lower-extremity complications: use of Semmes-Weinstein monofilaments. Phys Ther 1996; 76(1): 68-71.

25. Nang EEK, Khoo CM, Tai ES, et al. Is there a clear threshold for fasting plasma glucose that differentiates between those with and without neuropathy and chronic kidney disease? The Singapore prospective study program. Am $J$ Epidemiol 2009; 169: 1454-1462.

26. Herman WH, Pop-Busui R, Braffett BH, et al. Use of the Michigan Neuropathy Screening Instrument as a measure of distal symmetrical peripheral neuropathy in type 1 diabetes: results from the Diabetes Control and Complications Trial/Epidemiology of Diabetes Interventions and Complications. Diabet Med 2012; 29(7): 937-944.

27. Greene DA, Stevens MJ, Feldman EL. Diabetic neuropathy: scope of the syndrome. Am J Med 1999; 107(2B): 2S-8S.

28. Cheng YJ, Gregg EW, Kahn HS, et al. Peripheral insensate neuropathy - a tall problem for US adults? Am J Epidemio 2006; 164: 873-880.

29. Tesfaye S, Selvarajah D. The Eurodiab study: what has this taught us about diabetic peripheral neuropathy? Curr Diab Rep 2009; 9(6): 432-434.

30. Ylitalo $\mathrm{K}$, Sowers MF, Heeringa $\mathrm{S}$ Peripheral vascular disease and peripheral neuropathy in individuals with cardiometabolic clustering and obesity. Diabetes Care 2011; 34: 1642-1647.

31. Fantuzzi G. Adipose tissue, adipokines, and inflammation. J Allergy Clin Immunol 2005; 115(5): 911-919.

32. Papanas N, Ziegler D. Prediabetic neuropathy: does it exist? Curr Diab Rep 2012; 12: 376-383.

33. Guzik TJ, Mangalat D, Korbut R. Adipocytokines - novel link between inflammation and vascular function? J Physiol Pharmacol 2006; 57(4): 505-528.

34. Wildman RP, Muntner P, Reynolds K, McGinn AP, Rajpathak S, Wylie-Rosett J, Sowers MR. The obese without cardiometabolic risk factor clustering and the normal weight with cardiometabolic risk factor clustering: prevalence and correlates of 2 phenotypes among the US population (NHANES 1999-2004). Arch Intern Med 2008; 168(15): 1617-1624.

35. Lemieux S, Prud'homme D, Bouchard C, Tremblay A, Despres JP. A single threshold value of waist girth identifies normal-weight and overweight subjects with excess visceral adipose tissue. Am J Clin Nutr 1996; 64(5): 685-693.

36. Taylor RW, Keil D, Gold EJ, Williams SM, Goulding A. Body mass index, waist girth, and waist-to-hip ratio as indexes of total and regional adiposity in women: evaluation using receiver operating characteristic curves. Am J Clin Nutr 1998; 67: 44-49.

37. Bosy-Westphal A, Geisler C, Onur S, et al. Value of body fat mass vs anthropometric obesity indices in the assessment of metabolic risk factors. Int $J$ Obes 2006; 30: 475-483.

38. Verdú E, Ceballos D, Vilches JJ, Navarro $X$. Influence of aging on peripheral nerve function and regeneration. J Peripher Nerv Syst 2000; 5(4): 191-208.

39. Rivner MH, Swift TR, Malik K. Influence of age and height on nerve conduction. Muscle Nerve 2001; 24: 1134-1141.

40. Jensen MP, Chodroff MJ, Dworkin RH The impact of neuropathic pain on health-related quality of life: review and implications. Neurology 2007; 68(15): 1178-1182.

41. Gordon Smith A, Russell J, Feldman EL, et al. Lifestyle intervention for prediabetic neuropathy. Diabetes Care 2006; 29(6): 1294-1299.

42. Tavee J, Zhou L. Small fiber neuropathy: a burning problem. Cleve Clin J Med 2009; 76(5): 297-305.

43. Vinik A. The approach to the management of the patient with neuropathic pain. J Clin Endocriol Metabol 2010; 95(11): 4802-4811. 